\title{
Distribution and Incidence of Blood-Borne Infection among Blood Donors from Regional Transfusion Centers in Burkina Faso: A Comprehensive Study
}

\author{
Nicha Wongjarupong, ${ }^{1,2}$ Sharad Oli, ${ }^{1}$ Mahamoudou Sanou, ${ }^{3}$ Florencia Djigma, ${ }^{3}$ Alice Kiba Koumare, ${ }^{3}$ Albert T. Yonli, ${ }^{3}$ \\ Mohamed A. Hassan, ${ }^{1}$ Kristin Mara, ${ }^{4}$ William S. Harmsen, ${ }^{4}$ Terry Therneau, ${ }^{4}$ Oumar Barro, ${ }^{5}$ Ghislaine Vodounhessi, ${ }^{6}$ \\ Salam Sawadogo, ${ }^{6}$ Jean Christopher Chamcheu, ${ }^{7}$ Jacques Simpore, ${ }^{3}$ Lewis R. Roberts, ${ }^{1}$ and Bolni M. Nagalo ${ }^{3,5 *}$ \\ ${ }^{1}$ Gastroenterology and Hepatology, Mayo Clinic Rochester, Rochester, Minnesota; ${ }^{2}$ Department of Medicine, University of Minnesota, \\ Minneapolis, Minnesota; ${ }^{3}$ Centre de Recherche Biomoléculaire Pietro Annigoni (CERBA)/LABIOGENE, University of Ouaga I Joseph Ki Zerbo \\ (JKZ), Ouagadougou, Burkina Faso; ${ }^{4}$ Division of Biomedical Statistics and Informatics, Mayo Clinic Rochester, Rochester, Minnesota; ${ }^{5}$ Division of \\ Hematology and Medical Oncology, Mayo Clinic Arizona, Scottsdale, Arizona; ${ }^{6}$ Centre National de Transfusion sanguine du Burkina Faso (National \\ Center for Blood Transfusion in Burkina Faso), Ouagadougou, Burkina Faso; ${ }^{7}$ School of Basic Pharmaceutical and Toxicological Sciences, College \\ of Pharmacy, University of Louisiana at Monroe, Monroe, Louisiana
}

\begin{abstract}
There is a high prevalence of blood-borne infections in West Africa. This study sought to determine the seroprevalence of blood-borne infections, including hepatitis B virus (HBV), hepatitis C virus (HCV), HIV, and syphilis, in blood donors in Burkina Faso. Blood donors were recruited from 2009 to 2013 in four major cities in Burkina Faso of urban area (Ouagadougou) and rural area (Bobo Dioulasso, Fada N'Gourma, and Ouahigouya). Serology tests including hepatitis B surface antigen, anti-HCV, anti-HIV, and rapid plasma reagin test were used for screening and were confirmed with ELISA. Disease prevalence was calculated among first-time donors. Incidence and residual risk were calculated from repeat donors. There were 166,681 donors; $43,084 \mathrm{had} \geq 2$ donations. The overall seroprevalence of HBV, HCV, HIV, and syphilis were $13.4 \%, 6.9 \%, 2.1 \%$, and $2.4 \%$, respectively. The incidence rates (IRs) of HBV, HCV, HIV, and syphilis infection were $2,433,3,056,1,121$, and 1,287 per 100,000 person-years. There was lower seroprevalence of HBV and HCV in urban area than in rural area (12.9\% versus $14.0 \%, P<0.001$; and $5.9 \%$ versus $8.0 \%, P<0.001)$, and no difference in HIV (2.1\% versus $2.1 \%, P=0.25)$. The IRs of new HBV, HCV, HIV, and syphilis were 2.43, 3.06, 1.12, and 1.29 per 100,000 person-years, respectively. The residual risk was one per 268 donations for HBV, one per 181 donations for $\mathrm{HCV}$, and one per 1,480 donations for HIV, respectively. In conclusion, this comprehensive study from four blood donation sites in Burkina Faso showed high HBV and HCV seroprevalence and incidence with high residual risk from blood donation.
\end{abstract}

\section{INTRODUCTION}

Blood-borne infections, including viral hepatitis B (HBV), viral hepatitis $\mathrm{C}(\mathrm{HCV})$, and human immunodeficiency virus (HIV), continue to be serious public health challenges for countries in West Africa, including Burkina Faso, which have relatively high seroprevalence compared with the rest of the world. ${ }^{1,2}$

The risk of transfusion-transmitted infections remains high in Burkina Faso for several reasons. First, there is a high prevalence of blood-borne infections in the area, increasing the risk of transmission during the disease window period. The residual risk estimates the risk of infection during the window period. Although screening for HBV, HCV, HIV, and syphilis before blood transfusion is mandatory as per WHO recommendations, nucleic acid testing that is routinely used in developed countries is still not available in Burkina Faso. ${ }^{3}$ In addition, in Burkina Faso, there is a wide use of blood transfusion in the setting of high prevalence of malaria, leading to a high rate of blood-borne infection caused by transmission.

Blood donors' studies can help estimate the incidence, prevalence, and residual infection risk of these infections in the communities. There are currently limited data regarding the seroprevalence and incidence in the different parts of Burkina Faso, including a comparison between urban and rural areas. In the limited resource setting, it is essential to define the areas needing to use resources for screening and prevention measures.

This study aimed to evaluate the seroprevalence, incidence, and residual risk of HIV, HBV, HCV, and syphilis infection

* Address correspondence to Bolni M. Nagalo, Division of Hematology and Medical Oncology, Mayo Clinic Arizona, 13400 E Shea Blvd., Scottsdale, AZ 85259. E-mail: nagalo.bolni@mayo.edu among blood donors of Burkina Faso. We compared the differences in the seroprevalence between urban and rural areas based on the site of the donation center and to determine the trend of the seroprevalence and incidence during this 5-year study period.

\section{SUBJECTS AND METHODS}

Subjects. This study obtained the approval of the CERBA/ Saint Camille Ethics Committee, Ouagadougou, Burkina Faso. We included eligible blood donors from the four centers of Burkina Faso, including Ouagadougou, Bobo Dioulasso, Fada N'Gourma, and Ouahigouya from January 1, 2009 to December 31, 2013. Volunteer non-remunerated blood donors were all healthy subjects, aged 17-64 years, with a body weight of more than $50 \mathrm{~kg}$. Health officers administered a standard pre-donation questionnaire at each site. Donors who were tested positive for HBV, HCV, HIV, or syphilis at initial donation were not eligible for further blood donation.

We recorded data such as age, sex, date of donation, blood donation site, and serology screening results. We classified donors from Ouagadougou as urban area donors, whereas those from the three other sites (Bobo Dioulasso, Fada N'Gourma, and Ouahigouya) were classified as rural areas donors. The first-time donation was the first donation at the site for each donor since the study start date. Repeat donation was defined as two or more donations from the same individual during the study period.

Serological testing and sample analysis. HBV, HCV, HIV, and syphilis serology were screened in all blood donor samples. Hepatitis B surface antigen (HBsAg) and anti-HCV antibody were detected using Hepanostika HBsAg Ultra with sensitivity of $100 \%$ and specificity of $99.9 \%$ (Biomérieux, 
Boxtel, the Netherlands, and Hepanostika), and HCV Ultra with sensitivity of $100 \%$ and specificity of $66 \%$ (Beijing United Biomedical Co. Ltd., Beijing, China), respectively. Antibodies to HIV type 1 and 2 were screened by Vironostika HIV UniForm II Ag/Ab with sensitivity of $100 \%$ and specificity of 99.7\% (Biomérieux, Boxtel, the Netherlands). Syphilis was screened by the rapid plasma reagin test with sensitivity of $81.3 \%$ and specificity of $24.8 \%$ (Cypress Diagnostics, Langdorp, Belgium).

The positive screening samples underwent confirmation tests using a second ELISA (Bio-Rad, Marnes la Coquette, France). The presence of antibodies to Treponema pallidum was confirmed with a T. pallidum hemagglutination test (Cypress Diagnostics, Langdorp, Belgium). A result was considered positive if both the first and second tests were positive.

Seroprevalence, incidence, and residual risk calculation. Seroprevalence was calculated as percentages for each infection and coinfection. Incidence rates (IRs) were calculated for repeat donors who donated blood two or more times during the study period. In the subgroup analysis, patients were classified by their age at the time of the first donation. The IRs were calculated by dividing the number of incident cases in the study period by the total number of person-years. Person-years were generated from the summation of inter-donation intervals for all donors. The residual risk of infection transmission was calculated based on the previous study. ${ }^{4}$ Window periods of HBV, HCV, and HIV were 56, 66, and 26 days, respectively. ${ }^{4}$

Residual risk $=($ Incident rate $\times$ duration of window period $) / 365$

Statistical analysis. Data were analyzed with SAS 9.1 (SAS Institute, Cary, NC). Poisson regression methods were used to compare seroprevalence and IRs among different years, sex, and urban/rural area.

\section{RESULTS}

Patient characteristics. Overall, there were 166,681 donors in total, of whom 43,084/166,681 (25.8\%) were repeat donors (Table 1). There were 119,437/166,681 (71.7\%) male patients, with the majority aged $20-25$ years $(n=65,541$ / 166,$681 ; 39.3 \%$ ). Of the four sites, the majority of patients were from Ouagadougou ( $n=83,884 / 166,681 ; 50.3 \%)$.

Seroprevalence among the first-time blood donors. The overall seroprevalence of HBV, HCV, HIV, and syphilis infections for first-time blood donors was $13.4 \%, 6.9 \%, 2.1 \%$, and, $2.4 \%$, respectively. The highest seroprevalence of coinfection was HBV and HCV in 1,713/166,681 (1.0\%) donors. Other coinfections were less common, with 528/166,681 (0.3\%) donors with HBV-HIV, 561/166,681 (0.3\%) donors with HBV-syphilis, and $238 / 166,681(0.1 \%)$ donors with HCV-HIV coinfection. When comparing infection trends over 5 years, there were no clinically meaningful differences or consistent trends in the seroprevalence of HIV, HBV, HCV, or syphilis over the 2009 to 2013 time period (Table 2).

For all centers combined, the seroprevalence of HBV infection was highest in the 20- to 25-year age-group $(9,247 /$ $65,541,14.1 \%)$ and lowest in the 40 - to 60 -year age-group $(1,310 / 11,600,11.3 \%)$. HCV infection was highest in and lowest in the 25- to 40-year age-group (2,674/45,036, 5.9\%) (Table 3).

All the four centers share similarity in terms of age and gender of the donors (Table 1). Of the four centers, Fada N'Gourma had the highest HBV $(4,130 / 23,721,17.4 \%)$ and HCV $(2,255 / 23,721,9.5 \%)$ seroprevalence. HIV seroprevalence was highest in Ouahigouya (1,849/19,597, 2.9\%), and syphilis seroprevalence was highest in Ouagadougou $(2,427 /$ $83,884,2.9 \%)$. Ouagadougou had the lowest seroprevalence HCV whereas Bobo Dioulasso had the lowest seroprevalence of HBV. There was lower seroprevalence of HBV and HCV in urban (Ouagadougou) than in the rural areas $(10,819 / 83,884$, $12.9 \%$ versus $11,557 / 82,797,14.0 \%, P<0.001$; and 4,923 / $83,884,5.9 \%$ versus $6,603 / 82,797,8.0 \%, P<0.001$, respectively). There was no difference in HIV seroprevalence between residents of the urban and rural areas $(1,728 / 83,884$, $2.1 \%$ versus $1,773 / 82,797,2.1 \%, P=0.25)$. There was higher seroprevalence of syphilis in the urban area than in the rural area $(2,427 / 83,884,2.9 \%$ versus $1,629 / 82,797,2.0 \%, P<$ $0.001)$.

Incidence rates and calculated residual risk among the repeat blood donors. Among the 43,084 repeat donors, there were 1,682, 2,106, 2,106, and 893 new HBV, HCV, HIV, and syphilis cases, respectively, during the study period. The overall IRs of HBV, HCV, HIV, and syphilis infection were 2,434, 3,056, 1,121, and 1,287 per 100,000 person-years, respectively.

Male donors had higher IRs of all blood-borne infections than female donors, all with $P<0.001$. The incident rates IRs of HBV, HCV, HIV, and syphilis were 2,433, 3,319, 1,159, and 1,441 per 100,000 person-years in males and 2,434, 2,284, 1,008 , and 833 per 100,000 person-years in females,

TABLE 1

General characteristic blood donors and blood-borne infection seroprevalence by sites $(n=166,681)$

\begin{tabular}{|c|c|c|c|c|c|c|}
\hline \multirow[b]{2}{*}{ Characteristics } & \multirow[b]{2}{*}{ Total $(N=166,681)$} & \multirow{2}{*}{$\begin{array}{c}\text { Urban } \\
\begin{array}{c}\text { Ouagadougou } \\
(N=83,884)\end{array}\end{array}$} & \multicolumn{4}{|c|}{ Rural } \\
\hline & & & $\begin{array}{l}\text { Overall rural } \\
(N=82,797)\end{array}$ & $\begin{array}{l}\text { Bobo Dioulasso } \\
(N=39,479)\end{array}$ & $\begin{array}{l}\text { Fada N'Gourma } \\
\quad(N=23,721)\end{array}$ & $\begin{array}{l}\text { Ouahigouya } \\
(N=19,597)\end{array}$ \\
\hline Mean age (SD) & $25.1(7.8)$ & $26.1(8.3)$ & $24.2(7.2)$ & $23.6(6.8)$ & $25.3(8.0)$ & $24.0(6.8)$ \\
\hline Male, $n(\%)$ & $119,437(71.7)$ & $58,747(70.0)$ & $60,690(73.3)$ & $29,070(73.6)$ & $17,043(71.8)$ & $14,577(74.4)$ \\
\hline \multicolumn{7}{|c|}{ Number of donations, $n(\%)$} \\
\hline 1 & $123,597(74.2)$ & $66,012(78.7)$ & $57,585(69.5)$ & $27,514(69.7)$ & $16,761(70.7)$ & $13,310(67.9)$ \\
\hline 2 & $24,446(14.7)$ & $10,273(12.2)$ & $14,173(17.1)$ & $6,920(17.5)$ & $3,512(14.8)$ & $3,741(19.1)$ \\
\hline 3 & $8,579(5.1)$ & $3,258(3.9)$ & $5,321(6.4)$ & $2,403(6.1)$ & $1,621(6.8)$ & $1,297(6.6)$ \\
\hline 4 & $3,802(2.3)$ & $1,483(1.8)$ & $2,319(2.8)$ & $1,032(2.6)$ & 758 (3.2) & $529(2.7)$ \\
\hline$\geq 5$ & $6,257(3.8)$ & $2,858(3.4)$ & $3,399(4.1)$ & $1,610(4.1)$ & $1,069(4.5)$ & $720(3.7)$ \\
\hline \multicolumn{7}{|c|}{ Seroprevalence, $n(\%)$} \\
\hline HBV & $22,376(13.4)$ & $10,819(12.9)$ & $11,557(14.0)$ & $4,656(11.8)$ & $4,130(17.4)$ & $2,771(14.1)$ \\
\hline $\mathrm{HCV}$ & $11,535(6.9)$ & $4,932(5.9)$ & $6,603(8.0)$ & 2,499 (6.3) & $2,255(9.5)$ & $1,849(9.4)$ \\
\hline HIV & $3,501(2.1)$ & $1,728(2.1)$ & $1,773(2.1)$ & $663(1.7)$ & $550(2.3)$ & $560(2.9)$ \\
\hline Syphilis & $4,056(2.4)$ & $2,427(2.9)$ & $1,629(2.0)$ & $553(1.4)$ & $523(2.2)$ & $553(2.8)$ \\
\hline
\end{tabular}


TABLE 2

Seroprevalence of HBV, HCV, HIV, and syphilis among the first-time blood donor by first year of donation

\begin{tabular}{|c|c|c|c|c|c|c|}
\hline Distribution & $\begin{array}{c}\text { All }(n=166,681) \\
n(\%)\end{array}$ & $\begin{array}{c}2009(n=37,747) \\
n(\%)\end{array}$ & $\begin{array}{c}2010\left(\begin{array}{c}n=38,380) \\
n(\%)\end{array}\right. \\
(\%)\end{array}$ & $\begin{array}{c}2011\left(\begin{array}{c}n=32,879) \\
n(\%)\end{array}\right. \\
\end{array}$ & $\begin{array}{c}2012\left(\begin{array}{c}n=26,032) \\
n(\%)\end{array}\right.\end{array}$ & $\begin{array}{c}2013\left(\begin{array}{c}n=31,643) \\
n(\%)\end{array}\right. \\
\end{array}$ \\
\hline HBV & $22,376(13.4)$ & $4,880(12.9)$ & $5,234(13.6)$ & 4,448 (13.5) & 3,315 (12.7) & 4,499 (14.2) \\
\hline $\mathrm{HCV}$ & $11,535(6.9)$ & $2,361(6.3)$ & $2,511(6.5)$ & $2,590(7.9)$ & $1839(7.1)$ & $2,234(7.1)$ \\
\hline HIV & $3,501(2.1)$ & $677(1.8)$ & 776 (2.0) & $698(2.1)$ & $645(2.5)$ & 705 (2.2) \\
\hline Syphilis & $4,056(2.4)$ & 847 (2.2) & $603(1.6)$ & $1,367(4.2)$ & $692(2.7)$ & $547(1.7)$ \\
\hline
\end{tabular}

respectively, with $P$-value of $0.99,<0.001,0.098$, and $<0.001$, respectively. When classified by the year of initial donation, the incident rates were comparable from 2009 to 2012, with the highest IRs of all infections in 2013 compared with the other years for all four infections (Table 4). When classified by agegroup, the 25- to 40-age group had the highest IR of HBV, whereas the 20- to 25-age group had the highest IRs of HCV and syphilis, and the 40- to 60-age group had the highest IR of HIV. The 17- to 20-year age-group had the lowest IRs of IRs of HBV, HIV, and syphilis. The 40- to 60-year age-group had the lowest HCV IR. (Table 5).

The calculated residual risks were one per 268 donations for $\mathrm{HBV}$, one per 181 donations for HCV, and one per 1,480 donations for HIV, respectively.

\section{DISCUSSION}

This study is an extension of a previous study from our group assessing the prevalence and incidence of blood-borne infections in blood donors from three donation centers in 2009. ${ }^{5}$ With comprehensive data from four blood donation sites, our study showed that HBC and HCV seroprevalence, incidence, and residual risk of blood-borne infections among blood donors remain high in Burkina Faso.

Burkina Faso has a high seroprevalence of transfusion transmissible infections. The seroprevalence of HBV infection in our study is $13.4 \%$. Burkina Faso is among the countries with high HBV seroprevalence in West Africa, ranging from seroprevalence of $4.1-17.4 \% .^{2,6,7}$ The seroprevalence of HCV infection is $6.9 \%$ in this cohort, which is the fourth highest among West African countries below Mauritania, and Mali, and Ghana, respectively. ${ }^{2,6}$ HIV and syphilis seroprevalence in Burkina Faso is relatively comparable to that in other countries in West Africa. ${ }^{2}$ With limited healthcare access for diagnostic testing, treatment and limited vaccination for HBV, countries in West Africa continue to have high prevalence and incidence of HBV, HCV, and HIV.

During the 5 years of our study from 2009 to 2013, the seroprevalence of $\mathrm{HBV}, \mathrm{HCV}$, and HIV remained relatively stable. Comparing our data from the Ouagadougou center with the previously reported data in 2002, our study showed a lower seroprevalence of HBV and HIV. ${ }^{8}$ However, we found a similar $\mathrm{HCV}$ seroprevalence compared with $2002 .^{8}$ The latest data from 2015 to 2017 showed a lower seroprevalence of the three infections. ${ }^{4}$ This suggests a trend of gradually decreasing HBV, HCV, and HIV seroprevalence among donors. However, the seroprevalence of these transfusion-transmitted infectious diseases is still very high compared with that in the rest of the world.

Interestingly, the donors of $>40$ years age-group have a lower seroprevalence of HBV infections. The median age of HBV diagnosis in Burkina Faso is 32 years. ${ }^{9}$ There is a widespread annual screening of HBV infection during events such as the world hepatitis day in Burkina Faso. In addition, individuals who were tested positive at a younger age would be excluded from a donation. ${ }^{10}$ This could create the appearance of a lower seroprevalence rate in the older cohort. The seroprevalence of HCV was relatively similar across all the agegroups but significantly higher in the small group of donors aged $>60$ years. Although there is no certain birth year cutoff to define risk for HCV infection in sub-Saharan Africa, the lack of blood product infection screening in the earlier period is likely contributed to HCV infection in the older population. Previous systemic reviews of HCV infection in sub-Saharan Africa also showed a significant increase of $\mathrm{HCV}$ prevalence at 55 years and older. ${ }^{11}$

We found a higher seroprevalence of HBV and HCV infection in rural areas than in urban areas. This finding is consistent with

TABLE 3

Seroprevalence of HBV, HCV, HIV, and syphilis among the first-time blood donor by age group $(n=166,681)$

\begin{tabular}{|c|c|c|c|c|c|c|c|}
\hline \multirow[b]{2}{*}{ Prevalence } & \multicolumn{2}{|c|}{ Gender, $n(\%)$} & \multicolumn{5}{|c|}{ Age at first blood donation, $n(\%)$} \\
\hline & Male $(n=119,437)$ & Female $(n=47,244)$ & $>17$ to $20(n=44,426)$ & $>20$ to $25(n=65,541)$ & $>25$ to $40(n=45,036)$ & $>40$ to $60(n=11,600)$ & $>60(n=78)$ \\
\hline HBV (with any infection) & 17,758 (14.9) & $4,618(9.8)$ & $5,632(12.7)$ & $9,247(14.1)$ & $6,180(13.7)$ & $1,310(11.3 \%)$ & $7(9.0)$ \\
\hline HCV (with any infection) & $9,244(7.7)$ & $2,291(4.9)$ & $3,180(7.2)$ & $4,931(7.5)$ & $2,674(5.9)$ & $738(6.4)$ & $12(15.4)$ \\
\hline HIV (with any infection) & $2,551(2.1)$ & $950(2.0)$ & $782(1.8)$ & $1,345(2.1)$ & $1,033(2.3)$ & $339(2.9)$ & $2(2.6)$ \\
\hline Syphilis (with any infection) & $3,321(2.8)$ & $735(1.6)$ & $991(2.2)$ & $1778(2.7)$ & $1,015(2.3)$ & $270(2.3)$ & $2(2.6)$ \\
\hline \multicolumn{8}{|l|}{ Coinfection } \\
\hline HBV And HCV & $1,464(1.2)$ & $249(0.5)$ & $480(1.1)$ & $752(1.2)$ & $400(0.9)$ & $80(0.7)$ & $1(1.3)$ \\
\hline HBV And HIV & $412(0.3)$ & $116(0.3)$ & $117(0.3)$ & $217(0.3)$ & $162(0.4)$ & $32(0.3)$ & $0(0.0)$ \\
\hline HCV And HIV & $188(0.2)$ & $50(0.1)$ & $57(0.1)$ & $109(0.2)$ & $48(0.1)$ & $24(0.2)$ & $0(0.0)$ \\
\hline HBV And syphilis & $476(0.4)$ & $85(0.2)$ & $134(0.3)$ & $268(0.4)$ & $134(0.3)$ & $25(0.2)$ & $0(0.0)$ \\
\hline HCV And syphilis & $299(0.3)$ & $63(0.1)$ & $108(0.2)$ & $150(0.2)$ & $79(0.2)$ & $25(0.2)$ & $0(0.0)$ \\
\hline HIV And syphilis & $53(0.0)$ & $15(0.0)$ & $16(0.0)$ & $32(0.1)$ & $16(0.0)$ & $4(0.0)$ & $0(0.0)$ \\
\hline HBV, HCV, and syphilis & $66(0.1)$ & $6(0.0)$ & $16(0.0)$ & $34(0.1)$ & $20(0.0)$ & $2(0.0)$ & $0(0.0)$ \\
\hline HIV, HBV, HCV, and syphilis & $1(0.0)$ & $1(0.0)$ & $1(0.0)$ & $0(0.0)$ & $1(0.0)$ & $0(0.0)$ & $0(0.0)$ \\
\hline
\end{tabular}


TABLE 4

Incidence rates per 100,000 person-years of HBV, HCV, HIV, and syphilis among the repeated donors by year of first donation $(n=$ 43,084)

\begin{tabular}{lcrrrr}
\hline Distribution & 2009 & \multicolumn{1}{c}{2010} & 2011 & 2012 & 2013 \\
\hline HBV & $2,366.8$ & $2,445.7$ & $2,447.4$ & $2,560.6$ & $4,113.1$ \\
HCV & $3,056.4$ & $3,058.7$ & $2,800.8$ & $3,209.6$ & $5,398.0$ \\
HIV & $1,299.8$ & 852.4 & 945.1 & $1,328.3$ & $1,670.3$ \\
Syphilis & $1,133.7$ & $1,487.9$ & $1,543.4$ & 877.9 & $1,542.1$ \\
\hline \multicolumn{4}{l}{ HBV = hepatitis B virus; HCV = hepatitis C virus; HIV = human immunodeficiency virus. }
\end{tabular}

previous studies in Sierra Leone and Ethiopia. ${ }^{12,13}$ Rural areas have less access to health care, including the diseases diagnosis. There is more public knowledge in urban areas to assess donors' own risk for blood-borne disease infection and possibly exclude themselves from the donation. For HBV, the home newborn delivery, which is more common in rural areas, limits the mother-to-child transmission prevention and delays the first dose of HBV vaccination as one of the studies in rural West Burkina Faso found that $38.2 \%$ vaccinated children remains unprotected with a low HBV antibody level. ${ }^{14}$ In our study, HIV seroprevalence was similar between urban and rural areas, whereas in the previous studies in Burkina Faso and subSaharan Africa, HIV had a higher prevalence in urban areas. $^{15-17}$ However, HIV infection is also related to unprotected sex and has a strong reverse relationship to poverty. Our finding implicates increasing HIV seroprevalence in urbanizing rural areas.

The IRs of the repeat donors' infections were similar from 2009 to 2012, with a significant increase in infection rates in 2013. However, there was no change in the infection rates among the first-time donor prevalence. This is likely because donors in 2013 had a shorter follow-up period (less than 1 year) than those in the previous periods, which may have skewed the data.

Our study presents the most extensive report of blood donor data from Burkina Faso to date, including data from the four major blood donation sites (Ouagadougou, BoboDioulasso, Fada N'Gourma, and Ouahigouya) with a 5-year study period. There are several limitations in interpreting blood donor data to estimate the actual prevalence in the general population. Blood donors are generally healthy, so the data are likely to underestimate the general population disease. ${ }^{1,18}$ Besides, donors are male predominance and younger, which does not represent the general population.

\section{CONCLUSION}

Despite the availability of early detection testing for transfusion-transmitted infectious diseases, blood-borne infectious diseases continue to be a significant threat to safe blood transfusion in sub-Saharan Africa. Our study demonstrated that blood transfusion in Burkina Faso remains a substantial risk of transmission of HIV, and viral hepatitis. We found a relatively high

TABle 5

Incidence rates per 100,000 person-years of HBV, HCV, HIV, and syphilis among the repeated donors by age-group $(n=43,084)$

\begin{tabular}{lrrrrc}
\hline Distribution & $>17$ to 20 & $>20$ to 25 & $>25$ to 40 & $>40$ to 60 & $>60$ years \\
\hline HBV & $2,167.1$ & $2,546.3$ & $2,572.0$ & $2,483.2$ & 0 \\
HCV & $2,951.7$ & $3,380.9$ & $2,908.7$ & $2,250.4$ & 0 \\
HIV & 980.9 & $1,123.9$ & $1,230.3$ & $1,330.5$ & 0 \\
Syphilis & $1,042.6$ & $1,415.9$ & $1,363.2$ & $1,378.8$ & 0 \\
\multicolumn{7}{l}{ HBV = hepatitis B virus; HCV } \\
\multicolumn{7}{l}{ hepatitis C virus. }
\end{tabular}

seroprevalence, incidence, and residual risk of HBV, HCV, HIV, and syphilis infection among blood donors. Furthermore, there was a higher seroprevalence of HBV and HCV in rural areas versus urban areas and similar seroprevalence of HIV and syphilis in urban areas versus rural areas. The high IRs suggest a significant ongoing acquisition of new blood-borne infections among adults in the country. Therefore, a better blood safety strategy by selecting low-risk blood donors and using nucleic acid detection is needed to reduce the burden of transfusion-transmitted infectious diseases in Burkina Faso.

Received June 5, 2020. Accepted for publication December 20, 2020. Published online February 22, 2021.

Acknowledgments: We want to thank Eleonore Kafando and the staff of the National Transfusion Blood Center of Burkina Faso at Ouagadougou. We also thank the Mayo Clinic Department of Informatics, Mayo Clinic, and Rochester, MN, for analyzing the data.

Financial support: This work was supported by Mayo Clinic Institutional funding.

Authors' addresses: Nicha Wongjarupong, University of Minnesota, Minneapolis, MN, E-mail: nwongjarupong@gmail.com. Sharad Oli, Maimonides Medical Center, Brooklyn, NY, E-mail: drsharadoli@ gmail.com. Mahamoudou Sanou, Florencia Djigma, Alice Kiba Koumare, Albert T. Yonli, and Jacques Simpore, University of Ouaga I Joseph Ki Zerbo (JKZ), Ouagadougou, Burkina Faso, E-mails: mahamoudsanou@hotmail.com, florencia.djigma@gmail.com, alice_ kiba@yahoo.fr, yonlitheo@yahoo.fr, and jacques.simpore@yahoo.fr. Mohamed A. Hassan, University of Pittsburgh, Pittsburg, PA, E-mail: hassan.mohamed.dr@gmail.com. Kristin Mara, William S. Harmsen, Terry Therneau, and Lewis R. Roberts, Mayo Clinic Rochester, Rochester, MN, E-mails: mara.kristin@mayo.edu, harmsen.william@ mayo.edu, therneau@mayo.edu, and roberts.lewis@mayo.edu. Oumar Barro and Bolni M. Nagalo, Mayo Clinic Arizona, Phoenix, AZ, E-mails: barro.oumar@mayo.edu and nagalo.bolni@mayo.edu. Ghislaine Vodounhessi and Salam Sawadogo, National Center for Blood Transfusion in Burkina Faso, Ouagadougou, Burkina Faso, E-mails: ghislainetal11270@gmail.com and salemserein@hotmail.com. Jean Christopher Chamcheu, University of Louisiana at Monroe, Monroe, LA, E-mail: chamcheu@ulm.edu.

This is an open-access article distributed under the terms of the Creative Commons Attribution (CC-BY) License, which permits unrestricted use, distribution, and reproduction in any medium, provided the original author and source are credited.

\section{REFERENCES}

1. The Polaris Observatory Collaborators, 2018. Global prevalence, treatment, and prevention of hepatitis $B$ virus infection in 2016: a modelling study. Lancet Gastroenterol Hepatol 3: 383-403.

2. WHO, 2016. Global Status Report on Blood Safety and Availability. Geneva, Switzerland: World Health Organization. Available at: http://apps.who.int/iris/bitstream/handle/10665/ 254987/9789241565431-eng.pdf. Accessed May 1, 2020.

3. WHO, 2012. Blood Donor Selection: Guidelines on Assessing Donor Suitability for Blood Donation. Luxembourg City, Luxembourg: World Health Organization.

4. Yooda AP et al., 2019. Residual risk of HIV, HCV, and HBV transmission by blood transfusion between 2015 and 2017 at the regional blood transfusion center of Ouagadougou, Burkina Faso. J Blood Med 10: 53-58.

5. Nagalo BM et al., 2012. Seroprevalence and incidence of transfusion-transmitted infectious diseases among blood donors from regional blood transfusion centres in Burkina Faso, west Africa. Trop Med Int Health 17: 247-253.

6. Nkansah C, Serwaa D, Osei-Boakye F, Owusu-Ampomah R, 2019. Seroprevalence and trend of hepatitides among blood donors in a district hospital in Ghana: a nine-year retrospective, 
descriptive cross-sectional study. J Immunoassay Immunochem 41: 71-83.

7. Okoroiwu HU, Okafor IM, Asemota EA, Okpokam DC, 2018. Seroprevalence of transfusion-transmissible infections (HBV, $\mathrm{HCV}$, syphilis and HIV) among prospective blood donors in a tertiary health care facility in Calabar, Nigeria; an eleven years evaluation. BMC Public Health 18: 645.

8. Kania D, Sangare L, Sakande J, Koanda A, Nebie YK, Zerbo O, Combassere AW, Guissou IP, Rouet F, 2009. A new strategy to improve the cost-effectiveness of human immunodeficiency virus, hepatitis $B$ virus, hepatitis $C$ virus, and syphilis testing of blood donations in sub-Saharan Africa: a pilot study in Burkina Faso. Transfusion 49: 2237-2240.

9. Wongjarupong $\mathrm{N}$ et al., Characteristics of patients with chronic hepatitis $B$ virus infection with genotype $E$ predominance in Burkina Faso. Hepatol Commun 4: 1781-1792.

10. World Hepatitis Alliance, 2014. World Hepatitis Day 2014 Summary Report. Available at: https://www.worldhepatitisalliance.org/ world-hepatitis-day-2014-summary-report. Accessed May 1, 2020.

11. Mohd Hanafiah K, Groeger J, Flaxman AD, Wiersma ST, 2013. Global epidemiology of hepatitis $C$ virus infection: new estimates of age-specific antibody to HCV seroprevalence. Hepatology 57: 1333-1342.

12. Abdella $S$ et al., 2020. Sero-prevalence of transfusion transmittable infections: HIV, hepatitis B, C and Treponema pallidum and associated factors among blood donors in Ethiopia: a retrospective study. PLoS One 15: e0241086.

13. Yambasu EE, Reid A, Owiti P, Manzi M, Murray MJS, Edwin AK, 2018. Hidden dangers-prevalence of blood borne pathogens, hepatitis B, C, HIV and syphilis, among blood donors in Sierra Leone in 2016: opportunities for improvement: a retrospective, cross-sectional study. Pan Afr Med J 30: 44.

14. Sanou AM, Ilboudo AK, Meda CZ, Togozia A, Coulibaly A, Cisse A, Sagna T, Kania D, Tarnagda Z, 2018. Hepatitis B vaccination in Burkina Faso: prevalence of HBsAg carriage and immune response in children in the western region. $J$ Infect Dev Ctries 12: 1002-1008.

15. Magadi MA, 2013. The disproportionate high risk of HIV infection among the urban poor in sub-Saharan Africa. AIDS Behav 17: 1645-1654.

16. Zegeye EA, Mbonigaba J, Kaye S, Johns B, 2019. Assessing the cost of providing a prevention of mother-to-child transmission of HIV/AIDS service in Ethiopia: urban-rural health facilities setting. BMC Health Serv Res 19: 148.

17. Kirakoya-Samadoulougou F, Nagot N, Samadoulougou S, Sokey M, Guiré A, Sombié I, Meda N, 2016. Declining HIV prevalence in parallel with safer sex behaviors in Burkina Faso: evidence from surveillance and population-based surveys. Glob Health Sci Pract 4: 326-335.

18. Baha $W$ et al., 2013. Prevalence and risk factors of hepatitis $B$ and $C$ virus infections among the general population and blood donors in Morocco. BMC Public Health 13: 50. 